\title{
Gene Set Enrichment Analyses: lessons learned from the heart failure phenotype
}

Vinicius Tragante ${ }^{1 *}$ D, Johannes M. I. H. Gho ${ }^{1}$, Janine F. Felix², Ramachandran S. Vasan ${ }^{3}$, Nicholas L. Smith ${ }^{4}$, Benjamin F. Voight ${ }^{5,6,7}$, CHARGE Heart Failure Working Group, Colin Palmer ${ }^{8}$, Pim van der Harst ${ }^{9}$, Jason H. Moore ${ }^{10}$ and Folkert W. Asselbergs $s^{1,11,12,13}$

\author{
* Correspondence: \\ v.tragante@umcutrecht.nl \\ 'Department of Cardiology, Division \\ Heart \& Lungs, University Medical \\ Center Utrecht, Heidelberglaan 100, \\ 3584 CX Utrecht, The Netherlands \\ Full list of author information is \\ available at the end of the article
}

\begin{abstract}
Background: Genetic studies for complex diseases have predominantly discovered main effects at individual loci, but have not focused on genomic and environmental contexts important for a phenotype. Gene Set Enrichment Analysis (GSEA) aims to address this by identifying sets of genes or biological pathways contributing to a phenotype, through gene-gene interactions or other mechanisms, which are not the focus of conventional association methods.

Results: Approaches that utilize GSEA can now take input from array chips, either gene-centric or genome-wide, but are highly sensitive to study design, SNP selection and pruning strategies, SNP-to-gene mapping, and pathway definitions. Here, we present lessons learned from our experience with GSEA of heart failure, a particularly challenging phenotype due to its underlying heterogeneous etiology.

Conclusions: This case study shows that proper data handling is essential to avoid false-positive results. Well-defined pipelines for quality control are needed to avoid reporting spurious results using GSEA.
\end{abstract}

Keywords: Gene set enrichment analyses, Heart failure, Coronary artery disease

\section{() Biomed Central}

\section{Introduction}

Gene Set Enrichment Analysis (GSEA) is a statistical method to assess whether differences in expression of gene sets between two phenotypes are statistically significant $[1,2]$. It was initially designed for analysis of mRNA expression values, obtained from the then recently developed microarray technology [3], based on the observation that existing methods at the time were not capable of separating the small difference in expression profiles between two classes, and a grouping strategy was necessary. The original study [3] also developed collections of gene sets, based on biological knowledge available at the time.

Since then, multiple additional methods and reference gene sets have been developed, in an attempt to tackle caveats that emerged with the increase in use of GSEA and the new types of data, such as genome-wide association studies (GWAS) and exome sequencing. Among the current existing methods, Pathway Studio [4], MAGENTA [5], PANTHER [6], EVA [7] and Ingenuity [8] are commonly used, whereas the most common gene set definitions are Gene Ontology [9], KEGG [10, 11], REACTOME [12, 13], BIOCARTA [14] and MSIGdb [15].

(c) The Author(s). 2017 Open Access This article is distributed under the terms of the Creative Commons Attribution 4.0 International License (http://creativecommons.org/licenses/by/4.0/), which permits unrestricted use, distribution, and reproduction in any medium, provided you give appropriate credit to the original author(s) and the source, provide a link to the Creative Commons license, and indicate if changes were made. The Creative Commons Public Domain Dedication waiver (http://creativecommons.org/ publicdomain/zero/1.0/) applies to the data made available in this article, unless otherwise stated. 
Heart failure (HF) is a major medical problem of the Western world, carrying a high morbidity, mortality and economic burden $[16,17]$. The susceptibility to develop HF is thought to be partially genetically based [18], but despite a tremendous increase in knowledge regarding etiology and risk factors for HF, still relatively little is known about genetic factors related to HF incidence. Until now, genetic causes of HF have mainly been identified in rare cases of non-ischemic HF with monogenic inheritance [19]. Genetic studies for complex diseases, such as HF, focus on main effects of single loci using strict statistical thresholds for significance, and typically do not consider more complicated biological, genomic, or environmental hypotheses and models in their primary scans.

While genome-wide studies have succeeded in identifying a multitude of genetic variants affecting disease risk, for incident heart failure (HF) thus far only two SNPs have been identified in different ethnicities [20], most likely due to the relatively small sample sizes of the efforts so far, and the fact that HF is a very heterogeneous phenotype. Functional SNPs with small main effects may not replicate across studies due to context-dependent effects, such as the selection criteria of each cohort. Novel alternative analysis approaches to GWAS data that focus on the combined effects of many loci, each making a small contribution to overall disease susceptibility, such as GSEA, may provide a solution for the aforementioned limitations. Based on evidence from GSEA, SNPs may be selected for further studies even if the association of that SNP with heart failure is sub-genome-wide significant. Discovery of loci that contribute susceptibility to complex diseases like HF through gene-by-gene or gene-by-environment interactions may segregate main effects at the individual loci that are weak or even entirely absent, motivating approaches like GSEA or pathway-based methods that detect association at the biological systems level.

We hypothesize that multiple loci interact to contribute to development of HF. GSEA can be used to summarize genome-wide and exome array data integrating biochemical systems and gene function. With possible gene-gene interactions present in gene sets, potentially novel pathophysiological pathways can emerge, underlying the development of HF, which are missed by conventional methods.

Given the statistical approach of each method, the gene sets and the input each one takes, outputs obtained for a given phenotype may differ widely between GSEA methods, causing uncertainty on how to interpret the data and move research forward. Our goal in this paper is not to review each method separately (refer to Elbers et al. [21] for a broad comparison), but instead to offer general guidelines that can be applied to every method of the GSEA class. To illustrate these methods, we use genome-wide data from the CHARGE consortium [20], PREVEND [22, 23] and Go-DARTS [24] reporting incident HF, and CARDIoGRAM [25] and C4D [26] for coronary artery disease (CAD).

\section{Cohort descriptions}

\section{Discovery}

We use in this paper as input the results of the GWAS meta-analysis on incident heart failure performed by the CHARGE (Cohorts for Heart and Aging Research in Genomic Epidemiology) consortium [20]. The analysis of the CHARGE - Heart Failure Working Group, part of the CHARGE Consortium, included 4 prospective cohort studies: the 
Atherosclerosis Risk in Communities Study (ARIC), the Cardiovascular Health Study (CHS), the Framingham Heart Study (FHS) and the Rotterdam Study (RS). These studies included participants of European and African ancestry who were free of HF at study baseline. Incident HF cases were identified during follow-up by self-report, administrative data or periodic clinical in-study examinations. A total of 20,926 participants of European ancestry and 2895 participants of African ancestry with available genome wide data were eligible. The average age at baseline ranged from 53.3 to 72.6 years and $57 \%$ were women. In total $2526(12.1 \%)$ incident HF events were identified among those of European ancestry over an average of 11.5 years follow-up and $466(16.1 \%)$ during 13.7 years of follow up among those of African ancestry. The average age at the time of HF onset was 73.6 years and $52 \%$ of events occurred among women. In those with European and African ancestry, $73 \%$ and $78 \%$, respectively, had no history of myocardial infarction prior to the diagnosis of heart failure.

\section{Replication}

Firstly, we conducted replication using incident heart failure cases from the Genetics of Diabetes Audit and Research Study in Tayside Scotland (Go-DARTS) [24], a cohort with European individuals with type 2 diabetes, which was genotyped using Illumina ExomeChip. This chip gives us the advantage of having one-to-one SNP-to-gene mapping, because protein-coding SNPs were included in this chip, and their mapping is known. Secondly, we conducted replication using heart failure cases and GWAS data of the Prevention of REnal and Vascular ENdstage Disease (PREVEND) Study ( $n=3,418$, non-diabetics), a Dutch ongoing prospective study investigating the natural course of increased levels of urinary albumin excretion and its relation to renal and cardiovascular disease [22, 23].

All studies in CHARGE, Go-DARTS and PREVEND received institutional review board approval, and all participants provided written informed consent for the use of their DNA for research.

\section{Initial pathway results}

We analyzed the CHARGE [20] meta-analysis results for HF with EVA [7] using MSIGdb [15]. We defined $P<0.05$ as the threshold for significance, calculated via enrichment tests counting the number of SNPs below this threshold as compared to the total of SNPs mapped to each gene, in the first step. We mapped SNPs to genes using BEDtools [27], dbSNP142 and the RefSeq gene reference, both datasets downloaded from the UCSC Genome Browser [28], using a $500 \mathrm{~kb}$ window for mapping. We then used the obtained gene $p$-values to calculate pathway $p$-values, by counting the number of gene $p$-values below the significance threshold of 0.05 as a proportion of the number of genes in each pathway.

We followed exactly the same procedure and phenotype (HF) for the other two datasets (GoDARTS and PREVEND), with the exception of the SNP-to-gene mapping step for GoDARTS since all Exome chip SNPs are known to code specific genes. Additional file 1: Table S1 presents $p$-values for all significant pathways in the analysis of the CHARGE data and their $p$-values from the analyses of the other two cohorts. 
Four pathways were significant for all three studies: KEGG_TYPE_I_DIABETES_ MELLITUS, KEGG_ALLOGRAFT_REJECTION, KEGG_GRAFT_VERSUS_HOST_ DISEASE and KEGG_ASTHMA. These pathways share a considerable amount of SNPs (15 out of 30,43, 36 and 41, respectively), the vast majority of which is related to the MHC complex (Additional file 2: Figure S1). Permutation tests run in R [29] with 100000 simulations showed a low likelihood of these results arising by chance, with $P<10^{-06}$ for Graft versus Host disease, $P \sim 0.002$ for Type 1 Diabetes and Asthma and $P \sim 0.007$ for Allograft rejection (Additional file 1: Table S2).

Despite the multiple and convincing evidence in favor of the results, we explain below why these are false positives, which is mainly as a consequence of data handling. In the following sections we will explore strategies to avoid such false-positive findings in GSEA.

\section{Pre-processing the data}

Every GSEA method is highly dependent on the input provided. As an example, while PANTHER [6] accepts as input gene symbols without associated $p$-values, MAGENTA, Ingenuity and EVA take as input SNP IDs with $p$-values, obtained, for instance, from GWAS, and use this $p$-value information to calculate proportions of significant signals according to predefined thresholds.

When providing SNPs and $p$-values to GSEA, it is important to check for the LD structure of the data provided, since GWAS arrays and especially gene-centric arrays have dense coverage in regions of particular interest, and lower coverage in other regions of the genome. Such unbalance can lead to artificial enrichment of regions, in case a densely covered region presents an LD block under the $p$-value threshold determined; the opposite can also happen, and a potentially important region may be lost due to a non-significant LD block in the vicinity. A recent study by Sobota et al. [30] concludes that an $\mathrm{r}$ [2] of 0.3 is a reasonable threshold to eliminate redundancy, and our tests corroborate that recommendation, with the original 2,438,671 SNPs narrowed down to 410,986 , without losing any locus of the top SNP hits $\left(P<10^{-3}\right)$.

\section{SNP-to-gene mapping}

Another crucial step is the SNP-to-gene mapping. Recent studies suggest that a $100 \mathrm{~kb}$ window gives on average one mapping per SNP [31]. Using the same window of $100 \mathrm{~kb}$, we obtained 2.6 mappings per SNP on average, with 1,801,727 SNPs mapped $4,762,714$ times), at the cost of missing $\sim 600 \mathrm{k}$ SNPs, which are probably located in gene deserts.

Larger windows can unveil promoter and enhancer mappings, which are commonly within $500 \mathrm{~kb}$ regions of the SNP [32-34] (although enhancers can be found further away [35]). Also, more complex regions, such as MHC, may have SNPs in LD separated by over $3 \mathrm{M}$ bases [36], and rare variant effects may be found up to $2.5 \mathrm{M}$ bases away from the tag SNP $[37,38]$.. We do not recommend large windows for SNP-to-gene mapping, as the number of mappings becomes intractable: our tests showed an average of 9 mappings per SNP with a $500 \mathrm{~kb}$ window, with 2,241,172 SNPs being mapped 20,159,139 times. For a $1 \mathrm{Mb}$ window, 2,387,544 SNPs were mapped 34,793,184 times, giving an average of 14.6 mappings per SNP, most of them likely to be false mappings regarding LD structure (encompassing multiple unrelated genes, due to the mapping 
based only on distance). Such extensive mapping may lead to overplay of the effects of a single SNP in multiple genes, and if it happens to be a highly significant gene, it could drive the overrepresentation of a whole gene set [39]. These patterns remain even after LD clumping (best proxy method implemented in Plink [40], independent of $p$-value), with 301,509 SNPs mapped 830,835 times (average 2.8 mappings per SNP) for a $100 \mathrm{~kb}$ window, and 367,854 SNPs mapped 3,542,909 times (average 9.6 mappings per SNP) using a $500 \mathrm{~kb}$ window. A $100 \mathrm{~kb}$ window thus seems reasonable to obtain the most manageable ratio of "true" mappings, under an assumption that the nearest genes are most likely the ones affected by a variant (although it is arguably not always the case).

\section{Gene sets}

Efforts to represent collections of gene sets representing biological pathways have been an active research topic for the past 20 years $[9,41,42]$. In general terms, each pathway contains a set of genes that contribute to a certain metabolic process or biological function, obtained from a multitude of experiments. Each of these experiments and databases has its own level of confidence, which may lead to spurious results if not taken into account.

To explain this point, we describe an example. MSigdb [15] has collections of signature databases available that were composed in 2005, including KEGG [10]. The definition for Type I diabetes (T1D) from KEGG at MSigdb contains 44 genes, including 20 $H L A$ genes. While it is striking that so many HLA-related genes play a role in development of T1D at the same time, it is not surprising that inflammatory processes are involved in the pathogenesis of this disease. However, KEGG updated its definition of the T1D disease pathway with the most recent research findings, and it currently consists of 22 genes, only three of which are $H L A$ genes [43]. This new set is arguably more precise, and is likely to lead to different results when used as definition of a GSEA, although MSIGdb has not been updated since. Adriaens et al. [44] suggest that Reactome should be taken as an initial database for analyses, because of its curation system, which gives more reliable definitons. Other methods, such as Ingenuity [8], also have curation systems, to improve the confidence on the results.

Recently, Frost et al. [45] addressed the problem of generality of pathways, i.e., multiple genes in the same region are assigned to a pathway due to the knowledge one of these genes is involved in the process, but there is no certainty which. These researchers used gene expression data to score how significant each gene of a pathway definition actually is, and were able to narrow down pathways to represent more meaningful biological processes. We believe this is a necessary step to help reduce false positive findings in GSEA.

\section{Test case: CARDloGRAM and C4D}

In the GWAS field, the high number of false-positive findings of the early studies [46-48] has led to a very stringent $p$-value significance threshold and a mandatory replication step in independent samples $[49,50]$. We believe that the GWAS protocol should be used to GSEA as well. In order to test our method, we decided to use a well-established meta-analysis on CAD from the CARDIoGRAMplusC4D consortium [51]. Coronary ARtery DIsease Genome wide Replication and Metaanalysis (CARDIoGRAM) is a consortium of 14 cohorts with multiple recruitment 
criteria, assessing coronary artery disease (CAD) status of over 80,000 individuals of European ancestry. The Coronary Artery Disease Genetics (C4D) is a similar consortium, established to assess CAD status of over 30,000 patients from four different cohorts of European and south Asian ancestry. Results of independent metaanalyses from CARDIoGRAM [25] and C4D [26] are available online, with GWAS chip and imputed SNPs from CARDIoGRAM ( 2.5 M SNPs) but only GWAS chip results from C4D ( $500 \mathrm{k} \mathrm{SNPs})$. In order to level both datasets we performed imputation using DISTMIX [52] on C4D, and after imputation we had most of the same SNPs in both datasets. After 1000Genomes-based clumping of an unrelated phenotype, metabolic burden (methods described in Tragante et al. [53]) $\left(r^{2}>=0.3\right)$, both datasets had over 400,000 SNPs for pathway analysis. We used a $100 \mathrm{~kb}$ window around each SNP to map them to genes in the vicinity. Finally, we used a combination of MSigdb gene sets C2 (curated gene sets) and C5 (Gene Ontology gene sets), which include REACTOME, KEGG and Gene Ontology terms.

Using the full original CARDIoGRAM as the discovery set (given its bigger sample size), we obtained 224 pathways with $P<0.05,69$ pathways with $P<0.01$ and 13 pathways with $P<=0.001$. With C4D for replication, we obtained 21 of the 224 discovery pathways under the $P<0.05$ threshold, eight of those with $P<0.01$ and two below $P<=0.001$. With the clumped datasets, we obtained 250 pathways with $P<0.05,68$ pathways with $P<0.01$ and 15 pathways with $P<=0.001$. Replication in C4D reached 20 of 250 discovery pathways with $P<0.05$, nine of those with $P<0.01$ and three with $P<=0.001$. Moreover, eight pathways are significant for both CARDIoGRAM and C4D, original sets and clumped sets. The main pathway identified is Biocarta's Acute Myocardial Infarct pathway, which is an on-target result. Other pathways are related to lipid and platelet metabolisms, which are also directly related to CAD and MI as risk factors (Additional file 1: Table S3). One advantage of the clumped results over the original datasets is the convergence between gene $p$-values of both datasets. While for the original datasets 30 out of 56 bona fide genes, (i.e., with genome-wide significant SNPs) have either $P<0.05$ or $P>0.05$ on both datasets, for the clumped datasets, 38 out of these 56 genes are convergent (Additional file 1: Table S4).

\section{Rerunning the heart failure phenotype with complete preprocessing}

Using CHARGE GWAS and imputed data ( 2.5 M SNPs) as the discovery set (due to the bigger sample size), we identified 54 pathways with $P<0.01,3$ of these with $P<=0.001$ (Additional file 1: Table S5), which were the same three pathways that were significantly associated after FDR correction $(q=0.05)$.

We used two independent cohorts to validate our findings: GoDARTS ( $240 \mathrm{k}$ exome chip SNPs) and PREVEND (GWAS chip and imputed $~ 2.5 \mathrm{M}$ SNPs). We used the following parameters for discovery and replication: clumped SNP lists with the lead signal from an LD block of $\mathrm{r}^{2}>=0.3$, a $100 \mathrm{~kb}$ window for mapping SNPs to genes, and MSigdb gene sets C2 (curated gene sets) and C5 (Gene Ontology gene sets). Replication in PREVEND resulted in 33 pathways with $P<0.01,3$ of these with $P<0.001$ (Additional file 1: Table S6) and significant after FDR correction $(\mathrm{q}=0.05)$. However, there was no overlap between any of these pathways.

We then decided to increase our SNP-to-gene mapping window, to $500 \mathrm{~kb}$, keeping the other parameters the same. We then obtained 74 pathways with $P<0.01,19$ of 
those with $P<0.001$ (Additional file 1: Table S7) and significant after FDR correction ( $q=0.05$ ) with the CHARGE dataset, and 57 pathways with $P<0.01,10$ of those with $P<0.001$ (Additional file 1: Table S8) and significant after FDR correction $(\mathrm{q}=0.05)$. Two pathways overlapped between these two sets of results: NIKOLSKY_BREAST_ CANCER_16P13_AMPLICON, consisting of 120 genes, and NIKOLSKY_BREAST_ CANCER_8Q23_Q24_AMPLICON, consisting of 158 genes. We then investigated how many of these genes have low $p$-values for both datasets. Surprisingly, none of the 120 genes of the first pathway were significant in both datasets, and only three out of the 158 genes of the second pathway had a $P<0.05$ in the first pathway, which is lower than expected by chance (binomial $P=0.03$ ).

The possible advantage of the known SNP-to-gene mapping of the Exome Chip data in their use in GoDARTS is undermined by the fact that there are few SNPs per gene after clumping, leading to imprecise statistics per gene. Therefore, we could not make use of the Exome Chip results.

\section{Discussion}

Gene set enrichment analysis is a strategy to bring insight into biological mechanisms that lead to disease. Experience from years of GWAS analyses has shown that effect sizes of genetic variants identified are small. Grouping these variants into biologically meaningful pathways, such as is done by GSEA, seems to be a potential alternative to gain power and identify true associations. A very detailed setup is necessary, however, to obtain reliable and reproducible results. From our example, we have shown that false-positive results can be found and even replicated without proper data handling. It is important to have welldefined pipelines for quality control, in order to avoid publishing false-positives, reworking and delay of scientific development. We provide in Fig. 1 a diagram of an ideal GSE analysis, with all pre-processing steps we described in the paper.

The approach described proved successful for CAD/MI. Using CARDIoGRAM and C4D, two big consortia that provide detailed meta-analysis results, we were able to

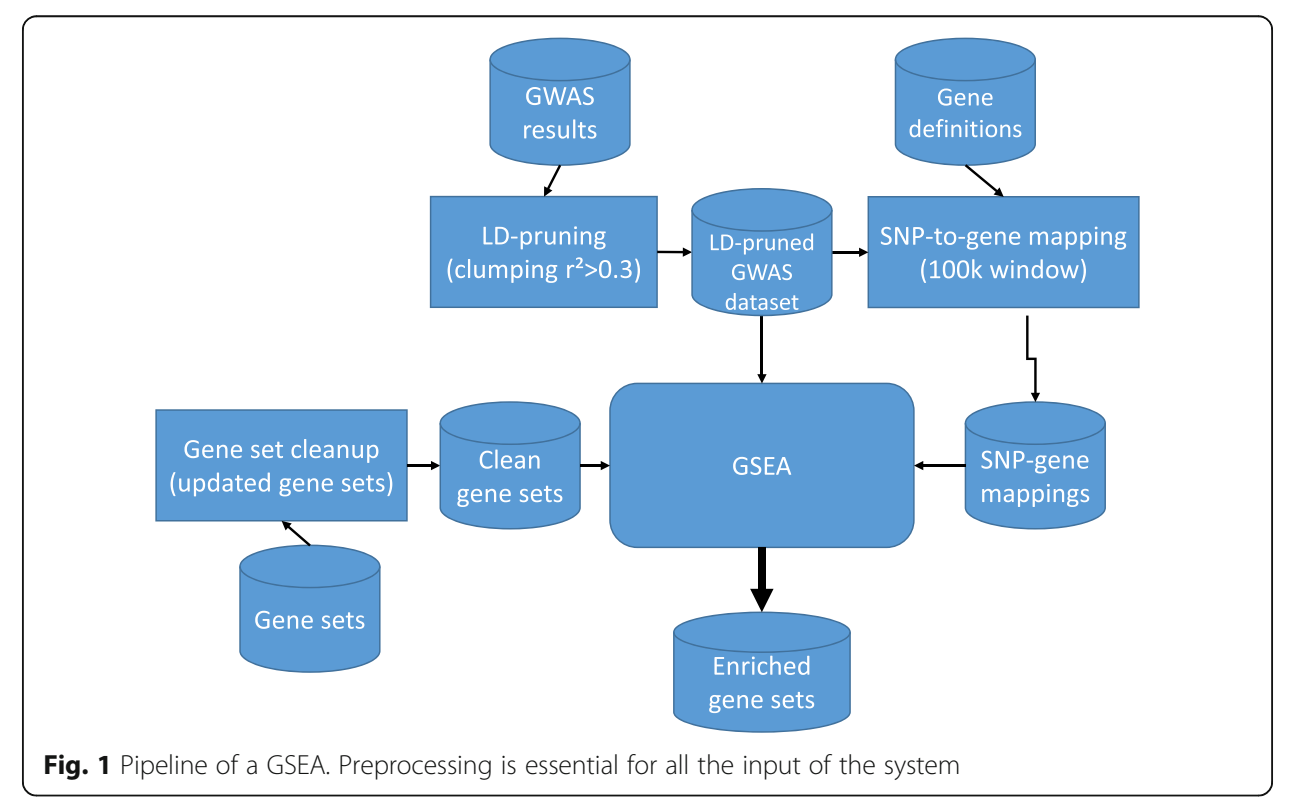


identify significant pathways that replicated on both datasets, and results are directly related to the phenotype. It is also worth noting that with the clumping, regions of high coverage of non-significant SNPs are cleaned up, giving a better estimate of the contribution of each gene in the phenotype. We exemplify this effect with the higher convergence rate between CARDIoGRAM and C4D for genes mapped from the genome-wide significant loci of the phenotype (38 out of 56, binomial $P=0.01$ ).

Several factors may have played a role in the absence of positive results for the heart failure phenotype after data QC. The phenotype definition is one of them. Given the complexity of the heart failure syndrome with different phenotypes between distinct ethnic cohorts and different etiologies, genome wide association studies on incident HF can be hampered by heterogeneity. For particularly heterogeneous phenotypes such as HF, large sample sizes are necessary to overcome the noise intrinsic to the data. Ideally, a better phenotyping and subsetting of the individuals might lead to a more clear separation of the genotypes, and results would be clearer with the sample sizes of current studies $\left(\sim 10^{5}-10^{6}\right.$ individuals). Of note, a similar GSEA setup in terms of SNP-togene mapping and mapping window size, conducted by Ghosh et al. [31], in the domain of coronary artery disease (CAD) (a less heterogeneous phenotype), with the aforementioned sample size $\left(\sim 10^{5}-10^{6}\right.$ individuals), succeeded in replicating pathways from Reactome, at a $P<0.05$ level. Their results led to new hypotheses on mechanisms of $\mathrm{CAD}$ that make biological sense demonstrating that a more homogeneous phenotype can lead to successful GSE analyses.

Furthermore, there is a predominance of immunological and cancer-related pathways among the pathways available in MSigdb, coming from multiple papers on the subcategory C2. Pathways with "cancer" as part of their names correspond to $9.4 \%$ of all pathways in this subcategory (446 out of 4725), and the terms "lymphoma", "myeloma" and "blastoma" bring an extra 268 results (5.7\%). A more balanced list of reference pathways may help identify biologically relevant processes for traits and diseases in other fields.

The SNP-to-gene mapping requires further improvement. In our tests, a window of $100 \mathrm{~kb}$ around the SNP position seems to provide the best ratio of mappings per SNP, in terms of biologically plausible mappings (lower windows would reduce the SNPs mapped up to over half of the input). We note, however, that this is not a final solution; methods that integrate functional assays with LD blocks could help narrow down the number of possible mappings, making the mapping more precise. Furthermore, there is the need to avoid multiple mappings of a single significant SNP to genes, as it could drive the overrepresentation of a whole gene set [39]. This mistake has led to retraction of a manuscript, since the main result had been inflated by the same SNP being mapped to eight genes in the same GO term [54]. This is a hypothesis to be investigated in the future, by making use of the current deluge of data being produced for functional analysis, such as ChIP-seq, RNA-seq, 4C-seq and eQTLs to provide a precise SNP-to-gene mapping and limit noise. Moreover, new computational methods that estimate the uncertainty of the potential causal, nearby gene(s) into the enrichment analysis could be very useful for appropriate significance assessment.

\section{Conclusions}

We believe that GSEA is particularly interesting, for example, in domains with high heritability and low penetrance, such as glucose levels, since multiple mechanisms may 
be influencing the outcome. It may also be useful for phenotypes in which the known genetic variants explain a low percentage of the phenotypic variance so far, such as blood pressure, because the individual effect of the SNPs associated is small, and grouping small effect SNPs can help in finding novel pathways. GSEA may also help identify cross-ethnic analyses, since different functional SNPs from the same gene without consistent effect across different populations may aggregate at the pathway level, making pathways more likely to replicate than individual SNPs.

GSEA methods have been gaining momentum as part of the GWAS discovery pipeline, and we believe that, with the appropriate setup and configuration, they will help elucidate biological mechanisms underlying phenotypes and diseases.

\title{
Additional files
}

Additional file 1: Table S2. Supplementary file. Supporting information and analyses results. (XLSX 45 kb)

Additional file 2: Figure S1. Overlapping genes among significant pathways for hearf failure before data processing. (PDF $145 \mathrm{~kb}$ )

\begin{abstract}
Abbreviations
4C-seq: Circularized chromosome conformation capture sequencing; C4D: The Coronary Artery Disease consortium; CAD: Coronary artery disease; CARDIoGRAM: Coronary Artery Dlsease Genome wide Replication and Meta-analysis; CHARGE: Cohorts for Heart and Aging Research in Genomic Epidemiology; ChIP-seq: chromatin immunoprecipitation sequencing; eQTL: Expression quantitative trait locis; GO: Gene ontology; GoDARTS: Genetics of Diabetes and Audit Research Tayside Study; GSEA: Gene set enrichment analysis; GWAS: Genome wide association study; HF: Heart failure; HLA: Human leukocyte antigen; KEGG: Kyoto Encyclopedia of Genes and Genomes; LD: Linkage disequilibrium; MAGENTA: Meta-Analysis Gene-set Enrichment of variant Associations; MHC: Major histocompatibility complex; MI: Myocardial infarction; MSigDB: Molecular Signatures Database; PANTHER: Protein Analysis Through Evolutionary Relationships; PREVEND: Prevention of Renal and Vascular Endstage Disease; RNA-seq: RNA sequencing (also known as whole transcriptome shotgun sequencing); SNP: Single nucleotide polymorphism
\end{abstract}

Acknowledgements

The authors acknowledge the department of Cardiology from UMC Utrecht for the support.

\section{Funding}

This work was supported by NIH R01 LM010098. Folkert W. Asselbergs is supported by a Dekker scholarship-Junior Staff Member 2014T001 - Netherlands Heart Foundation, UCL Hospitals NIHR Biomedical Research Centre, and by a clinical fellowship from the Netherlands Organization for Health Research (ZonMw grant 90700342). The research leading to these results has received funding from the European Union Seventh Framework Programme FP7/20072013 under grant agreement n HEALTHF22013601456 (CVgenesattarget).

\section{Availability of data and materials}

Please contact author for data requests.

\section{Authors' contributions}

$\mathrm{VT}$ conducted preprocessing of the data and enrichment analysis. JMIHG interpreted clinically results. Manuscript written by VT and JMIHG, with suggestions, corrections and reviews by JFF, RSV, NLS, BFV, CP, PVdH, JHM and FWA. JFF, RSV, NLS, CP and PvdH contributed with summary-level cohort data. All authors read and approved the final manuscript.

\section{Competing interests \\ The authors declare that they have no competing interests.}

Consent for publication

Not applicable.

Ethics approval and consent to participate

All data used in this project has been collected by third parties and been approved by each own Ethics committee.

\section{Publisher's Note}

Springer Nature remains neutral with regard to jurisdictional claims in published maps and institutional affiliations.

\section{Author details}

${ }^{1}$ Department of Cardiology, Division Heart \& Lungs, University Medical Center Utrecht, Heidelberglaan 100, 3584 CX

Utrecht, The Netherlands. '2Department of Epidemiology, Erasmus MC, University Medical Center Rotterdam, 
Rotterdam, The Netherlands. ${ }^{3}$ Departments of Medicine and Preventive Medicine, Boston University School of Medicine, Boston, MA, USA. ${ }^{4}$ Department of Epidemiology, University of Washington, Seattle, WA, USA. ${ }^{5}$ Department of Systems Pharmacology and Translational Therapeutics, Perelman School of Medicine, University of Pennsylvania, Philadelphia, PA, USA. ${ }^{6}$ Department of Genetics, Perelman School of Medicine, University of Pennsylvania, Philadelphia, PA, USA. IInstitute for Translational Medicine and Therapeutics, Perelman School of Medicine, University of Pennsylvania, Philadelphia, PA, USA. ${ }^{8}$ Population Pharmacogenetics Group, University of Dundee, Dundee, UK. ${ }^{9}$ Department of Cardiology, University Medical Center Groningen, University of Groningen, Groningen, The Netherlands. ${ }^{10}$ Department of Biostatistics and Epidemiology, Institute for Biomedical Informatics, Perelman School of Medicine, University of Pennsylvania, Philadelphia, PA, USA. ${ }^{11}$ Durrer Center for Cardiovascular Research, ICIN-Netherlands Heart Institute, Utrecht, The Netherlands. ${ }^{12}$ Institute of Cardiovascular Science, Faculty of Population Health Sciences, University College London, London, UK. ${ }^{13}$ Farr Institute of Health Informatics Research and Institute of Health Informatics, University College London, London, UK.

Received: 6 December 2016 Accepted: 9 May 2017

\section{Published online: 26 May 2017}

\section{References}

1. Shi J, Walker MG. Gene set enrichment analysis (GSEA) for interpreting gene expression profiles. Curr Bioinforma. 2007:2:133-7.

2. Subramanian A, Kuehn H, Gould J, Tamayo P, Mesirov JP. GSEA-P: a desktop application for gene Set enrichment analysis. Bioinformatics. 2007;23:3251-3.

3. Subramanian A, et al. Gene set enrichment analysis: a knowledge-based approach for interpreting genome-wide expression profiles. Proc Natl Acad Sci. 2005;102:15545-50. doi:10.1073/pnas.0506580102.

4. Nikitin A, Egorov S, Daraselia N, Mazo I. Pathway studio-the analysis and navigation of molecular networks. Bioinformatics. 2003;19:2155-7.

5. Segrè AV, et al. Common inherited variation in mitochondrial genes is not enriched for associations with type 2 diabetes or related glycemic traits. PLoS Genet. 2010;6:e1001058.

6. Mi H, Muruganujan A, Casagrande JT, Thomas PD. Large-scale gene function analysis with the PANTHER classification system. Nat Protoc. 2013;8:1551-66.

7. Reif DM, Dudek SM, Shaffer CM, Wang J, Moore JH. Exploratory Visual Analysis of Pharmacogenomic Results. Pacific Symposium on Biocomputing. 2005;10:296-307.

8. Krämer A, Green J, Pollard J, Tugendreich S. Causal Analysis Approaches in Ingenuity Pathway Analysis (IPA). Bioinformatics. 2014;30(4):523-30.

9. Ashburner M, et al. Gene ontology: tool for the unification of biology. Nat Genet. 2000;25:25-9.

10. Kanehisa M, Goto S. KEGG: kyoto encyclopedia of genes and genomes. Nucleic Acids Res. 2000;28:27-30.

11. Kanehisa M, Goto S, Sato Y, Furumichi M, Tanabe M. KEGG for integration and interpretation of large-scale molecular data sets. Nucleic acids research. 2012;40:D109-D114.

12. Joshi-Tope G, et al. Reactome: a knowledgebase of biological pathways. Nucleic Acids Res. 2005;33:D428-32.

13. Croft D, et al. The reactome pathway knowledgebase. Nucleic Acids Res. 2014;42:D472-7.

14. Nishimura D. BioCarta. Biotech Softw Int Rep. 2001;2:117-20.

15. Liberzon A, et al. Molecular signatures database (MSigDB) 3.0. Bioinformatics. 2011;27:1739-40.

16. Mosterd A, Hoes AW. Clinical epidemiology of heart failure. Heart. 2007;93:1137-46.

17. Yancy CW, et al. 2013 ACCF/AHA guideline for the management of heart failure: a report of the american college of cardiology foundation/american heart association task force on practice guidelines. J Am Coll Cardiol. 2013;62:e147-239.

18. Lee DS, et al. Association of parental heart failure with risk of heart failure in offspring. N Engl J Med. 2006;355:138-47.

19. Arbustini $E$, et al. The MOGE (S) classification for a phenotype-genotype nomenclature of cardiomyopathy: endorsed by the World Heart Federation. J Am Coll Cardiol. 2013;62:2046-72.

20. Smith NL, et al. Association of genome-wide variation with the risk of incident heart failure in adults of european and african ancestry: a prospective meta-analysis from the cohorts for heart and aging research in genomic epidemiology (CHARGE) consortium. Circ Cardiovasc Genet. 2010;3:256-66. doi:10.1161/ circgenetics.109.895763.

21. Elbers CC, et al. Using genome-wide pathway analysis to unravel the etiology of complex diseases. Genet Epidemiol. 2009;33:419-31.

22. Pinto-Sietsma SJ, et al. Urinary albumin excretion is associated with renal functional abnormalities in a nondiabetic population. J Am Soc Nephrol. 2000;11:1882-8.

23. Hillege $\mathrm{HL}$, et al. Urinary albumin excretion predicts cardiovascular and noncardiovascular mortality in general population. Circulation. 2002;106:1777-82.

24. Doney AS, et al. The FTO gene is associated with an atherogenic lipid profile and myocardial infarction in patients with type 2 diabetes a genetics of diabetes audit and research study in tayside scotland (Go-DARTS) study. Circ Cardiovasc Genet. 2009;2:255-9.

25. Preuss $\mathrm{M}$, et al. Design of the coronary ARtery Dlsease genome-wide replication and meta-analysis (CARDloGRAM) study a genome-wide association meta-analysis involving more than 22000 cases and 60000 controls. Circ Cardiovasc Genet. 2010;3:475-83.

26. Coronary Artery Disease Genetics Consortium. A genome-wide association study in europeans and south asians identifies five new loci for coronary artery disease. Nat Genet. 2011;43:339-44.

27. Quinlan AR, Hall IM. BEDTools: a flexible suite of utilities for comparing genomic features. Bioinformatics. 2010;26:841-2. doi:10.1093/bioinformatics/btq033.

28. Karolchik, D., Hinrichs, A. S. \& Kent, W. J. The UCSC Genome Browser. Current protocols in bioinformatics/editora board, Andreas D. Baxevanis ... [et al.] Chapter 1, Unit1 4, doi:10.1002/0471250953.bi0104s40 (2012). 
29. Ihaka R, Gentleman R. R: a language for data analysis and graphics. J Comput Graph Stat. 1996;5:299-314.

30. Sobota RS, et al. Addressing population-specific multiple testing burdens in genetic association studies. Ann Hum Genet. 2015;79:136-47.

31. Ghosh $\mathrm{S}$, et al. Systems genetics analysis of genome-wide association study reveals novel associations between Key biological processes and coronary artery disease. Arteriosclerosis, thrombosis, and vascular biology. ATVBAHA. 2015:115:305513.

32. Wang K, Li M, Bucan M. Pathway-based approaches for analysis of genomewide association studies. Am J Hum Genet. 2007:81:1278-83. doi:10.1086/522374.

33. Daly MJ, Rioux JD, Schaffner SF, Hudson TJ, Lander ES. High-resolution haplotype structure in the human genome. Nat Genet. 2001;29:229-32.

34. Barrett JC, Fry B, Maller J, Daly MJ. Haploview: analysis and visualization of LD and haplotype maps. Bioinformatics. 2005;21:263-5.

35. Visel A, Rubin EM, Pennacchio LA. Genomic views of distant-acting enhancers. Nature. 2009:461:199-205.

36. Stenzel A, et al. Patterns of linkage disequilibrium in the MHC region on human chromosome $6 \mathrm{p}$. Hum Genet. 2004;114:377-85.

37. Dickson SP, Wang K, Krantz I, Hakonarson H, Goldstein DB. Rare variants create synthetic genome-wide associations. PLoS Biol. 2010;8:e1000294. doi:10.1371/journal.pbio.1000294.

38. Wang $\mathrm{K}$, et al. Interpretation of association signals and identification of causal variants from genome-wide association studies. Am J Hum Genet. 2010;86:730-42. doi:10.1016/.j.jhg.2010.04.003.

39. Sedeño-Cortés AE, Pavlidis P. Pitfalls in the application of gene-set analysis to genetics studies. Trends Genet. 2014;30:513-4. doi:10.1016/j.tig.2014.10.001.

40. Purcell $\mathrm{S}$, et al. PLINK: a tool set for whole-genome association and population-based linkage analyses. Am J Hum Genet. 2007;81:559-75.

41. Cary MP, Bader GD, Sander C. Pathway information for systems biology. FEBS Lett. 2005;579:1815-20.

42. Kanehisa M. Toward pathway engineering: a new database of genetic and molecular pathways. Sci Technol Jpn. 1996;59:34-8.

43. KEGG. http://www.genome.jp/dbget-bin/www_bget?ds:H00408 . Accessed on 12 Oct 2015

44. Adriaens ME, et al. The public road to high-quality curated biological pathways. Drug Discov Today. 2008;13:856-62

45. Frost HR, Moore JH. Optimization of gene set annotations via entropy minimization over variable clusters (EMVC). Bioinformatics, btu110. 2014.

46. Ioannidis JP. Why most published research findings are false. Chance. 2005;18:40-7.

47. Chabris CF. et al. Most reported genetic associations with general intelligence are probably false positives. Psychological science. 2012. Doi: 10.1177/0956797611435528

48. Cantor RM, Lange K, Sinsheimer JS. Prioritizing GWAS results: a review of statistical methods and recommendations for their application. Am J Hum Genet. 2010;86:6-22.

49. Barsh GS, Copenhaver GP, Gibson G, Williams SM. Guidelines for genome-wide association studies. PLoS Genet. 2012;8:e1002812

50. Ioannidis JP, et al. Assessment of cumulative evidence on genetic associations: interim guidelines. Int J Epidemiol. 2008:37:120-32

51. Deloukas P, et al. Large-scale association analysis identifies new risk loci for coronary artery disease. Nat Genet. 2013:45:25-33.

52. Lee D, et al. DISTMIX: direct imputation of summary statistics for unmeasured SNPs from mixed ethnicity cohorts. Bioinformatics. 2015;31:3099-104.

53. Tragante, V. et al. Harnessing publicly available genetic data to prioritize lipid modifying therapeutic targets for prevention of coronary heart disease based on dysglycemic risk. Human genetics. 2016;135(5):453-467.

54. Dixson L. et al. Retraction for Dixson et al., Identification of gene ontologies linked to prefrontal-hippocampal functional coupling in the human brain. Proc Nat Acad Sci U S A. 2014;111(26): 9657-9662.

\section{Submit your next manuscript to BioMed Central and we will help you at every step:}

- We accept pre-submission inquiries

- Our selector tool helps you to find the most relevant journal

- We provide round the clock customer support

- Convenient online submission

- Thorough peer review

- Inclusion in PubMed and all major indexing services

- Maximum visibility for your research

Submit your manuscript at www.biomedcentral.com/submit 